\title{
Mit Kompass entscheiden
}

Flugzeugbau mit Kunststoffen, handliche Smartphones, deren Rechenleistung in den 60er-Jahren für Mondmissionen gereicht hätte vieles, was vor wenigen Jahrzehnten schwer vorstellbar war, lockt heute niemanden mehr hinter dem Ofen hervor. Heute bringen wir Werkstoffen und Maschinen das Kommunizieren bei, und ehrfürchtig erwarten wir die ersten Quantenrechner, die die gesamte heute verfügbare Rechenkapazität auf einer Maschine übersichtlichen Formats konzentrieren.

Unsere auf Wachstum ausgelegte Wirtschaft fordert den technischen Fortschritt, und wir liefern ihn. Mit jeder Forschungsarbeit, mit jeder neu entwickelten Technik. Und damit legen wir auch das Fundament der Welt, in der wir morgen leben.

Der Stifterverband hat im Dezember Zahlen zu den Forschungs- und Entwicklungsausgaben deutscher Unternehmen vorgelegt. Demnach hat die Wirtschaft 2016 mit 62,9 Milliarden Euro 3,1 Prozent mehr als im Vorjahr in den technischen Fortschritt investiert. Das ist erfreu- lich, sichern die Investitionen doch auch unsere Arbeitsplätze in einer sich stetig wandelnden Welt. Besonders investitionsfreudig ist übrigens die Automobilindustrie mit internen Ausgaben von 21,9 Milliarden Euro für Forschung und Entwicklung.

In erster Linie werden Investitionen nach unternehmerischen Kriterien entschieden: Wer investiert, möchte später Rendite erlangen. Doch die gesamte Tragweite der Entscheidungen geht über das Unternehmerische hinaus: Jeder in die Forschung investierte Euro ist eine Entscheidung für eine bestimmte Zukunft und gegen viele mögliche andere. Wir entscheiden aktiv, in welcher Zukunft wir leben werden.

Letzte Prognosen lassen auch in diesem Jahr deutschland- und auch weltweit eine stabile Wirtschaft, und damit rege Investitionen in Forschung und Entwicklung erwarten. Ich hoffe auf neue Technik, die günstig ist, dem Menschen nützt und sich mit der Umwelt verträgt. Für die zahlreichen anstehenden Weichenstellungen wünsche ich allen Entscheidern einen guten inneren Kompass. 4 